\title{
Avaliação participativa da segurança alimentar e nutricional em uma comunidade de Salvador, Brasil
}

\author{
Participatory food and nutrition security assessment in a \\ community of Salvador, Brazil
}

Marie Agnes Aliaga (https://orcid.org/0000-0001-5518-6912) ${ }^{1}$

Mirella Santos Ribeiro (https://orcid.org/0000-0001-8789-8367) ${ }^{1}$

Sandra Maria Chaves dos Santos (https://orcid.org/0000-0002-4706-0284) ${ }^{2}$

Leny Alves Bomfim Trad (https://orcid.org/0000-0002-8762-4320) ${ }^{1}$

${ }^{1}$ Instituto de Saúde Coletiva Universidade Federal da Bahia (UFBA). R. Basílio da Gama, Canela. 40110-040

Salvador BA Brasil.

marie.agnes.aliaga@ gmail.com

${ }^{2}$ Escola de Nutrição, UFBA.

Salvador BA Brasil.

\begin{abstract}
This paper addresses a Food and Nutrition Security (FNS) participatory assessment developed together with community leaders and residents in Salvador city, Bahia, Brazil. Our reflection aims to analyze this research - including design, data generated, and its use - discussing the concept of FNS and its existing assessment methods. Secondary data were found to be diffcult to access or of little utility to local activists. The household survey designed and used by the participants characterized the food and nutrition insecurity situation in vulnerable areas of the community, in a dialogue with national and socioeconomic indicators, evidencing robust data. First of all, the relevance of participatory approaches stood out: while the results show how much FNS is intricately embedded into a broader social vulnerability context, they show how critical it is to consider FNS research as a political instrument and the knowledge it produces as a power-related instrument. In this sense, FNS assessment is established as an emancipatory process, indissociable from the action and social change actors.
\end{abstract}

Key words Food and nutrition security, Social vulnerability, Community-based participatory research
Resumo Este artigo focaliza uma avaliação participativa da situação de Segurança Alimentar e Nutricional (SAN) desenvolvida junto a moradores e líderes comunitários em Salvador, Bahia. A reflexão visa analisar esta pesquisa - incluindo desenho, dados geridos e sua utilização - dialogando com o conceito de SAN e os métodos existentes da sua avaliação. Observou-se que os dados secundários são de difícil acesso elou pouco uteis para a militância local. Quanto à pesquisa domiciliar desenhada e utilizada pelos participantes, ela permitiu caracterizar o quadro de insegurança alimentar e nutricional em áreas vulneráveis da comunidade, dialogando com os indicadores nacionais e os dados socioeconômicos, demonstrando a robusteza dos dados. Antes de tudo, é a relevância de abordagens participativas que mais se destaca: no mesmo movimento em que os resultados mostram o quanto a questão da SAN se insere no quadro de vulnerabilidade social, eles evidenciam o quão necessário se faz pensar a pesquisa em SAN como instrumento político e o conhecimento gerido como instrumento do poder. Nesse sentido, a avaliação da situação de SAN se constitui como processo emancipatório, indissociável da ação e dos atores da transformação social.

Palavras-chave Segurança alimentar e nutricional, Vulnerabilidade social, Pesquisa participativa baseada na comunidade 


\section{Introdução}

O conceito de segurança alimentar, nascido no contexto das penúrias pós-segunda guerra mundial, ganhou corpo no final do século XX, passando a incorporar questões relativas ao acesso econômico e físico a alimentos, aos aspectos nutricionais e à qualidade dos alimentos, aos aspectos culturais sobre alimentação, assim como questões relativas à soberania alimentar, entre outros ${ }^{1}$. Com isso, o Comitê da Segurança Alimentar Mundial da Organização das Nações Unidas para Alimentação e Agricultura (CFS/ FAO) recomenda utilizar o conceito de Segurança Alimentar e Nutricional (SAN), o qual:

[...] existe quando todas as pessoas têm, a todo momento, acesso físico, social e econômico a uma alimentação segura, consumida em quantidade e qualidade suficiente, que cumpre com as suas necessidades nutricionais e preferências alimentares, em um ambiente com saneamento e serviços de saúde adequados, permitindo uma vida sadia e ativa ${ }^{2}$.

Com o compromisso dos países em avaliar e garantir a SAN para suas populações, notadamente a partir da assinatura da Declaração de Roma em 1996 por 186 deles, colocou-se a questão de saber como melhor analisar e entender o quadro de insegurança alimentar e nutricional ${ }^{3}$. $\mathrm{Na}$ tentativa de abarcar a multiplicidade de fatores que implicam no quadro de segurança alimentar e nutricional, foram desenvolvidos vários métodos de avaliação da SAN. Segundo Pérez -Escamilla e Segall-Corrêa ${ }^{4}$, há cinco métodos de avaliação comumente empregados em inquéritos nacionais: 1) método da FAO de cálculo da disponibilidade calórica diária per capita; 2) cálculo da renda mínima para consumo alimentar e não alimentar; 3) cálculo do consumo alimentar como os recordatórios quantitativos das últimas 24 horas, frequência de consumo alimentar ou a quantificação dos gastos familiares com aquisição de alimentos; 4) antropometria e; 5) escalas psicométricas do acesso familiar aos alimentos.

No seu estudo sobre a existência e a tipologia das avaliações em SAN nos países signatários da Declaração de Roma, Aliaga ${ }^{5}$ mostra que são utilizados instrumentos que vão desde previsões meteorológicas, medidas de produção agrícola e estudos de mercado, até indicadores nutricionais, de acordo com as realidades com a qual dialogam e as necessidades que a elas correspondem. Todos os países, porém, têm um ponto comum na avaliação da SAN: são poucos os documentos de avaliação que contam com a participação da sociedade civil local dentro da sua elaboração.
No Brasil, o suplemento "Segurança Alimentar" da Pesquisa Nacional por Amostra de Domicílios (PNAD) adotou a Escala Brasileira de Segurança Alimentar (Ebia), que se define como escala psicométrica do acesso familiar aos alimentos composta por 14 perguntas medindo a percepção das famílias em relação ao acesso aos alimentos ${ }^{6}$. A Pesquisa de Orçamento Familiar (POF), por sua vez, comporta estudos de aquisição e consumo alimentar e avaliação nutricional da população ${ }^{7-10}$. Há também avaliações locais da situação de segurança alimentar e nutricional, em determinadas comunidades ou em determinado grupo populacional, a maioria delas utilizando a Ebia e/ou avaliação antropométrica ${ }^{11-20}$. Outros utilizam grupos focais ou entrevistas em profundidade ${ }^{21,22}$. Outros ainda utilizam dados secundários para construir protocolos de avaliação municipal da $\mathrm{SAN}^{23}$.

No entanto, são pouquíssimos os estudos que envolvem os membros da comunidade investigada no desenho metodológico e na aplicação da pesquisa e/ou na análise dos seus resultados. Enquanto o Sistema Nacional de Segurança Alimentar e Nutricional (Sisan), criado em 2006, aposta fortemente na participação social da sociedade civil brasileira para o enfrentamento da insegurança alimentar e nutricional ${ }^{24}$, parece contraditória a pouca implicação das comunidades nos processos de pesquisa acerca da sua própria situação de SAN. Decerto, essa pouca implicação não contribui para combater o cenário brasileiro de baixa inserção do conceito de SAN e de pouco reconhecimento do Direito Humano à Alimentação Adequada (DHAA), ambos identificados como barreiras importantes da participação social em SAN ${ }^{25-30}$.

Argumenta-se, neste artigo, que é preciso colocar os instrumentos do conhecimento em SAN na mão das populações que sofrem de insegurança alimentar e nutricional e de lideranças ou organizações da sociedade civil que lutam no enfrentamento da vulnerabilidade social nas suas comunidades.

O primeiro elemento que sustenta tal argumento é a complexidade abarcada pela questão de como medir a SAN, chamando pela inserção dos processos de pesquisa nas dinâmicas sociais, políticas, econômicas e ambientais locais para o melhor entendimento da situação e dos processos de vulnerabilização nos quais se configura. $\mathrm{O}$ segundo elemento diz respeito à necessidade em situar os indivíduos como sujeitos políticos, construindo seus próprios saberes, para poder elaborar estratégias de enfrentamento e reinvindicação dos seus direitos. 
Esta abordagem é consistente com os princípios que fundaram as pesquisas participativas, que buscam implicar nelas os atores como agentes, donos dos seus próprios saberes, capazes de analisar seus problemas e de desenhar as suas próprias soluções ${ }^{31}$. Dentro das pesquisas participativas, a pesquisa-ação se constitui como a colocação de um instrumento de investigação e ação a disposição dos grupos sociais vulneráveis ${ }^{32}$, tentando "colocar o controle do saber nas mãos dos grupos e das coletividades que expressam uma aprendizagem coletiva tanto na sua tomada de consciência como no seu comprometimento com a ação coletiva" ${ }^{33}$.

Este artigo focaliza uma das primeiras ações empreendida dentro de um projeto de pesquisa -ação em SAN desenvolvido junto a líderes e moradores de uma região de Salvador, Bahia. Tratase de uma pesquisa domiciliar que visou avaliar a situação da SAN em áreas vulneráveis da região, com base em uma metodologia desenvolvida pelos participantes da pesquisa-ação, e cujos resultados poderiam sustentar as ações de militância dos mesmos. O presente artigo visa analisar essa experiência de pesquisa participativa em SAN, desde o seu desenho até os resultados gerados e a sua utilização pelos participantes, dialogando com os dados e com outras formas adotadas para avaliação em SAN.

\section{Os instrumentos da avaliação: uma construção participativa}

Pau da Lima é o nome dado a uma Região Administrativa, subdivisão do município de Salvador, que contava com 233.274 habitantes em $2010^{34}$. A formação do grupo de participantes da pesquisa-ação em foco se deu a partir de uma primeira oficina, organizada em fevereiro $2014 \mathrm{com}$ líderes comunitários e moradores pertencendo a uma rede existente de associações e igrejas. A oficina reuniu um total de 37 participantes da comunidade e teve como objetivo provocar uma reflexão acerca da situação de segurança alimentar e nutricional na região e também do próprio conceito de SAN. Com esta reflexão, pretendia-se desencadear a mobilização dos participantes e o desenho das primeiras ações do projeto.

Assim, foram formados grupos de trabalho, um dos quais ficou responsável pela definição do método de avaliação da situação da SAN na comunidade e organização da mesma. As reuniões do grupo foram semanais, entre maio e outubro 2014, com a participação de 5 pessoas da comunidade, entre eles dois moradores e uma mora- dora, assim como dois líderes comunitários, todos com idades entre 29 e 61 anos. As discussões incluíram os seguintes pontos: (1) protocolo de indicadores baseados em dados secundários de domínio público; (2) bases de dados municipais que desagregam dados por bairro ou distrito; (3) metodologias desenvolvidas e instrumentos de coleta utilizados na PNAD, e nas diferentes partes da POF, e discussão sobre métodos qualitativos; e (4) discussão e debate sobre a situação de SAN na comunidade, sobre métodos de coleta de informação e desafios enfrentados por pesquisas na comunidade.

Foram dois os instrumentos desenvolvidos para a avaliação de SAN na comunidade.

Primeiro, foi adaptado, para o nível local, um protocolo de indicadores municipais de SAN que utiliza dados secundários ${ }^{23,35}$, sendo coletados os indicadores ou indicadores similares desagregados no âmbito da Região, do Distrito Sanitário, ou, se estes não forem encontrados, relativos aos bairros. Os dados foram coletados, principalmente, nas bases de dados do Sistema de Informação Municipal de Salvador ${ }^{34}$, e do Sistema "TABNET" da Secretaria Municipal de Saúde de Salvador ${ }^{36}$.

A primeira observação nessa etapa dos trabalhos diz respeito à complexidade dos múltiplos bancos de dados, pouco accessíveis para membros da comunidade que não tem domínio de tais ferramentas. Além disso, vários dos indicadores locais não estão disponíveis nas bases de dados online e, quando estão, aplicam-se a delimitações geográficas diferentes (região administrativa, distrito sanitário, bairros), muitas vezes com nível de agregação que não dá conta da situação encontrada nas áreas mais vulneráveis. Por fim, alguns dos indicadores identificados tinham os seus valores mais recentes coletados no ano 2000.

Em geral, observou-se certa dissonância entre indicadores desatualizados, incompletos e agregados e, mas também uma realidade altamente heterogênea, dentro de um mesmo bairro ou de uma mesma região, e bastante dinâmica, com crescimento populacional exponencial e mobilidade populacional alta nas últimas décadas. Dados mais atuais e complementares, tais como indicadores nutricionais ou número de famílias registradas no cadastro único, podem, em teoria, ser acessíveis por pedido ao Distrito sanitário ou à subprefeitura. Estes dados foram solicitados às instâncias locais por líderes ao longo do projeto, o que levou a se defrontarem com a complexidade em obtê-los, apesar de alguns deles trabalharem na subprefeitura ou como agente comunitário de saúde. 
Esta situação mostra a dificuldade de acesso a dados públicos, atuais, simples de interpretar e de qualidade, para os moradores ou líderes da comunidade, contribuindo, em parte, para a pouca adesão dos participantes nesta etapa dos trabalhos.

Segundo, foi desenhada e aplicada uma pesquisa domiciliar de avaliação da SAN, com os seguintes objetivos: (1) avaliar a situação da SAN em uma área mais vulnerável da comunidade, como base para formulação de projetos e reivindicação da garantia do DHAA por parte das lideranças; (2) oferecer uma avaliação nutricional antropométrica para os moradores desta área; $\mathrm{e}$ (3) informar sobre a SAN e o DHAA, mobilizar para reivindicação do DHAA e convidar para a participação no projeto.

Foi aplicado um questionário de perguntas fechadas a um dos responsáveis em todos os domicílios, focalizando a percepção do entrevistado, tanto em relação ao seu acesso a alimentos, quanto em relação ao seu consumo. A escolha do grupo foi focalizar estas duas dimensões por serem consideradas as mais problemáticas na comunidade. $\mathrm{O}$ foco sobre a percepção do entrevistado foi, antes de tudo, motivado pela compreensão de que seria relevante realizar uma pesquisa domiciliar que não somente confirmaria a situação de insegurança alimentar e nutricional, mas também se aproximaria do entendimento dos moradores acerca do assunto e, não menos importante, tentaria despertar interesse pela questão da promoção da SAN e reivindicação do DHAA por parte dos moradores pesquisados, implicando-os na reflexão acerca do tema.

Com isso, o questionário foi complementado por perguntas abertas em $1 / 10$ dos domicílios, focando, primeiro, sobre o porquê da falta de alimentos no passado e da preocupação em faltar alimentos no futuro e, segundo, sobre o significado da alimentação saudável e as razões que levam os moradores a terem, ou não, segundo eles, alimentação saudável em casa. Os questionários foram acompanhados da avaliação nutricional dos moradores e de uma discussão sobre a SAN e o DHAA em cada domicílio, com convite para participar do projeto.

A pesquisa domiciliar foi realizada de novembro de 2014 a janeiro de 2015, em uma amostragem aleatória de 450 domicílios selecionados dentro da coorte de estudo do Centro de Pesquisa Gonçalo Muniz da Fundação Oswaldo Cruz Bahia (CPqGM-Fiocruz), que vem desenvolvendo neste território projetos desde 2001.

Para a análise dos dados quantitativos foram utilizadas estatísticas descritivas das vari- áveis socioeconômicas, de acesso e de consumo de alimentos, e de avaliação nutricional. Posteriormente, foi analisada a relação entre as variáveis socioeconômicas e os indicadores da SAN a partir de uma análise de tendências e de testes Qui-quadrado e Qui-quadrado de tendências. As análises foram realizadas utilizando o Epi-Info versão 7. As respostas às perguntas abertas foram gravadas, transcritas e analisadas por meio da criação de categorias analíticas emergindo das narrativas dos moradores. Foram redigidos cadernos de campo para registrar e sistematizar as observações da equipe de pesquisa.

A pesquisa domiciliar foi aprovada pelo Comitê de ética da instituição de pesquisa responsável pela pesquisa, e foi financiada pelo Conselho Nacional de Desenvolvimento Científico e Tecnológico (CNPq) junto ao Ministério do Desenvolvimento Social (MDS).

\section{Os resultados da avaliação: expressões da insegurança alimentar e nutricional}

A pesquisa domiciliar mostrou que $31 \%$ dos responsáveis por domicílios consultados afirmaram ter se preocupado com a falta de comida no mês anterior à pesquisa, e $17 \%$ afirmaram ter efetivamente faltado comida, incluindo $14 \%$ dos domicílios em que houve redução quantitativa de comida. A título de comparação, o estado da Bahia contava com $15,9 \%$ de domicílios em insegurança alimentar moderada ou grave em 2013, apontando por redução quantitativa de alimentos e/ou ruptura nos padrões de alimentação resultante da falta de alimentos nos três meses anteriores à pesquisa ${ }^{6}$. Em outros termos, na comunidade pesquisada, a proporção de domicílios relatando redução quantitativa de alimentos aproximou-se, em um mês, da proporção atingida no Estado em um período de três meses, lembrando que o estado mostrava taxas preocupantes de insegurança alimentar e nutricional.

Ressalta-se ainda que 273 (80\%) dos responsáveis afirmaram, muitas vezes com ênfase, ter a preocupação com a falta de comida para as próximas gerações. Entende-se que o fator preocupação para o futuro tem um papel importante no cotidiano dos moradores da comunidade, espelhando-se em uma situação de alta precariedade social e econômica.

Tanto os relatos dos moradores quanto a análise dos dados socioeconômicos identificaram a renda baixa e o desemprego como principais razões da falta de comida e da preocupação em faltar. A análise confirma uma associação estatisticamente significante entre essas variáveis. Alguns 
depoimentos indicam outro ponto de interesse: a dependência das mulheres em relação aos homens. De fato, a falta de alimento foi várias vezes atribuída, pela mãe de família, ao desemprego do marido ou do filho. A análise dos dados confirmou a grande diferença de acesso à comida entre os domicílios chefiados por mulheres e os por homens, com associação estatisticamente significante entre o gênero do responsável pelo domicílio e o relato, pelo mesmo, da preocupação em faltar comida, ou da falta efetiva de comida.

Os resultados mostram que a insegurança alimentar e nutricional da comunidade se insere em um contexto mais amplo de vulnerabilidade social, em linha com os resultados de estudiosos do tema ${ }^{6,37,38}$. De fato, os grupos que apresentam insegurança alimentar também são aqueles que estão expostos a vários outros fatores que caracterizam uma situação ou um estado de vulnerabilidade, entre eles: condições socioeconômicas deficitárias, meio ambiente degradante, escolaridade baixa, moradores de periferias urbanas, desemprego, entre outros. Dessa forma, as famílias que estão mais expostas à insegurança alimentar também são as que convivem com muitos outros indicadores de vulnerabilidade social ${ }^{39}$.

Coerentemente com a falta relatada de acesso à alimentação adequada, os resultados das avaliações antropométricas mostram que a comunidade sofre da chamada dupla carga ${ }^{40}$, convivendo tanto com a desnutrição quanto com o excesso de peso.

Entre os adultos, $6,2 \%$ da amostra mostravam déficit de peso, sendo que nas zonas urbanas da região Nordeste a prevalência encontrada foi de $2,3 \%$ para homens e $4,5 \%$ para mulheres em 2008-20097. Da mesma maneira, foi registrada uma prevalência de $20,9 \%$ de déficit de peso em idosos na comunidade, enquanto as prevalências estimadas para o Brasil inteiro são de 2,1\% a 4,4\% nesta faixa etária, em $2008-2009^{7}$. No mesmo tempo, enquanto as prevalências de obesidade foram estimadas em 11,5\% para homens e 15,6\% para mulheres nas zonas urbanas da região ${ }^{7}$, na comunidade pesquisada os resultados mostram uma prevalência de $22 \%$ de obesidade, incluindo casos de obesidade grau III, nos adultos. No final, somente $35 \%$ dos moradores adultos e idosos avaliados foram considerados com peso ideal, evidenciando um quadro preocupante de déficit de peso e obesidade.

Os resultados da avaliação nutricional mostram a inexistência de indicadores de desnutrição crônica nas crianças menores de 5 anos da comunidade. No entanto, há indicadores de desnu- trição aguda, reveladores de uma situação mais recente e/ou pontual: os dados apontam para um emagrecimento em um total de $7 \%$ das crianças, com magreza acentuada em 5\%. Segundo a Pesquisa Nacional de Demografia e Saúde da Criança e da Mulher (PNDS) ${ }^{41}$, déficits de peso em relação à altura são indicativos de casos agudos de desnutrição quando sua frequência ultrapassa 2 a 3\%. A título de comparação, em 2006, os resultados da PNDS mostraram um déficit de peso em relação à altura em apenas 1,9\% das crianças brasileiras menores de 5 anos, não ultrapassando $3 \%$ em qualquer estrato da populaçã $0^{41}$. Ressalta-se ainda, na mesma faixa etária, a prevalência de $7 \%$ de sobrepeso, e $1 \%$ de obesidade segundo este mesmo indicador nutricional, por um total de $8 \%$ de excesso de peso nos termos da PNDS, que mostrou uma prevalência de 6,6\% no Brasil em $2006^{41}$.

No geral, os dados mostram um quadro de magreza e obesidade preocupantes nas crianças e nos adolescentes da comunidade, com especial atenção para os dados de desnutrição aguda nas crianças menores de 5 anos e nos adolescentes entre 10 e 18 anos.

É importante ressaltar que as estratégias de sobrevivência das famílias não incluem recorrer ao Estado ou a Instituições: mesmo entre os domicílios que registraram falta de comida ou se preocuparam em faltar comida, poucos recorrem a doações de alimentos da família, vizinhos ou instituições, e não houve referência ao Governo ou a qualquer outra Instituição ao se abordar a questão da garantia da alimentação do domicílio.

Enquanto isso, surgiram diversas referências à Deus, que aparece como o que decide sobre o amanhã e também o que protege da falta de alimentos. Quando há responsabilidade de alguém para a garantia dos alimentos, no depoimento dos entrevistados, aquela responsabilidade é do próprio morador: Falta de responsabilidade minha! (E.3, Pergunta 1); Só quando eu morrer! Enquanto eu tiver braço pra trabalhar não falta não, viu?! Porque eu vou correr atrás! (E.3, Pergunta 2); Não sou aposentada, então eu tenho que trabalhar, me virar pra poder se manter (E.12, Pergunta 2). A aceitação e a responsabilização espontânea de si mesmo para a garantia de comida dentro de casa parecem predominar como indicado em formulações do tipo "a gente dá um jeito", ter que "se virar".

Também foi observado nos entrevistados certo desconforto ao falar da falta de comida, especialmente em domicílios nos quais esse fato já aconteceu, apontando para um estigma que 
persevera na medida em que a alimentação não é reconhecida como um direito, no discurso dos moradores. Assim, a responsabilização se torna individual e penaliza o responsável pelo domicílio: a falta de comida estaria testemunhando o fracasso do responsável em garantir alimentação dentro do domicílio. Este processo, pelo qual os sujeitos se tornam os culpados por seus próprios problemas de saúde, que na verdade são originários ou influenciados por fatores sociais, culturais e financeiros, é muito comum, e descrito por Vasconcelos ${ }^{42}$ como "culpabilização da vítima". Sendo a alimentação uma necessidade básica de sobrevivência, a sua falta constitui uma violação da dignidade humana ${ }^{43}$, alimentando a vergonha e a culpa no discurso dos entrevistados, e inibindo a mobilização social onde ela mais se faz necessária.

\section{A guisa de considerações finais: repensando a avaliação em SAN}

A pesquisa desenvolvida junto a líderes e moradores da comunidade investigada permitiu caracterizar o quadro de insegurança alimentar e nutricional em áreas vulneráveis da comunidade, dialogando com os indicadores nacionais e determinantes sociais da SAN observados em outros estudos. Isso demonstra certa robusteza dos dados obtidos: apesar de utilizar um instrumento de avaliação não padronizado, a pesquisa domiciliar conseguiu captar um fenômeno, o da insegurança alimentar e nutricional tal como foi concebida pelos participantes do projeto, o qual dialoga com a realidade social na qual se insere.

Situar a insegurança alimentar e nutricional no contexto mais amplo da vulnerabilidade social, como sugerem os resultados da pesquisa, permite entendê-la na sua dualidade individuocoletivo, apreendendo suas dimensões históricas, sociais, ambientais e políticas. Este tipo de abordagem tem duas principais implicações no que tange à avaliação da situação de SAN.

A primeira implicação diz respeito ao próprio fenômeno da insegurança alimentar e nutricional, à sua complexidade e abrangência, apontando para a necessidade em se repensar as metodologias para a sua análise.

A insegurança alimentar e nutricional vai muito além das suas expressões biológicas, abarcando as questões econômica, social, ambiental e cultural, que terminam por estabelecer elos de ligação entre a vida individual e a coletiva dos sujeitos $^{44}$. Na sua proposta por uma epistemologia política, Porto et al. ${ }^{45}$ apontam pela necessidade de formas de produção de conhecimento que reconheçam problemas de saúde e sua determinação socioambiental como complexos e multidimensionais, e que problematizem processos de vulnerabilização antes de situações de vulnerabilidade, determinação socioambiental antes de determinantes socioambientais.

Em particular, é problemático, na determinação social da saúde, o "fosso existente entre o plano macroestrutural e coletivo, e as condições e potencialidades dos sujeitos em planos mais pessoais e comunitários vinculados ao cotidiano e ao lugar" ${ }^{45}$, pois, segundo os autores:

A perspectiva biomédica e epidemiológica produziu uma racionalidade para as intervenções da sociedade e do Estado nos moldes de uma ciência normal, restringindo a dimensão social na determinação do processo saúde-doença ao plano instrumental dos atributos, com a sociedade sendo entendida como um agregado de indivíduos com características quantificáveis. Tal racionalidade não prioriza nem articula melhorias das condições de vida e trabalho com as estruturas sociais da sociedade $[. . .]^{45}$.

$\mathrm{Na}$ determinação social da insegurança alimentar e nutricional como na determinação social da saúde, é preciso construir enfoques de análise "críticos e transformadores", nos termos dos autores. Como base para essa reflexão, há o reconhecimento dos limites de métodos de avaliação da SAN que focalizam a dimensão individual ou domiciliar, que privilegiam aspectos fisiológicos ou biológicos quantificáveis, ou ain$\mathrm{da}$, que não abordem a insegurança alimentar e nutricional como um processo - o qual pode durar meses ou anos - e revelam um sujeito muitas vezes já adoecido.

Hoje, no Brasil, a SAN é principalmente medida através de instrumentos e indicadores que focalizam a dimensão domiciliar, como a Ebia e a pesquisa de orçamento familiar, e a dimensão individual, tal como a avaliação antropométrica dos indivíduos, esses indicadores sendo muitas vezes associados a variáveis socioeconômicas igualmente individuais ou domiciliares. Neste processo, corre-se o risco de deslocar o sujeito e/ ou grupo avaliado de um contexto de relações na qual se insere, reforçando o discurso pautado na sua responsabilização e em questões referentes, apenas, às suas práticas individuais. São nestas relações que as questões políticas estão presentes, inclusive aquelas que estão ligadas a ações de responsabilidade do Estado, em relação à SAN como objeto de direito humano. Em particular, importa ressaltar que a insegurança/segurança alimentar está diretamente relacionada ao acesso 
a serviços que são, em grande parte, dependentes da atuação do Estado (saneamento, fornecimento de água tratada, esgoto apropriado, serviços de saúde etc. $)^{38}$.

É preciso valorizar abordagens que, além da construção de indicadores individuais ou domiciliares, crie um espaço, tanto na academia quanto nas próprias comunidades, para se discutir o lugar da SAN nas relações entre o indivíduo e o coletivo, e para se pensar a responsabilidade do Estado.

A presente pesquisa domiciliar interveio no início da pesquisa-ação, em um momento em que os significados associados à SAN pelos participantes ainda estavam pautados na responsabilidade do sujeito, com pouco reconhecimento da alimentação enquanto direito, o que decerto impactou na escolha e na construção dos instrumentos da pesquisa. A incipiente familiarização entre os participantes, tanto com o conceito quanto com metodologias de pesquisa participativa, decerto contribuiu para que o desenho da pesquisa domiciliar desse um lugar de importância aos instrumentos já sendo utilizados no Brasil. Mesmo assim, a implicação dos moradores e dos líderes na construção metodológica e na análise dos resultados propiciou uma reflexão fértil a respeito da responsabilidade do Estado, e de como a insegurança alimentar e nutricional da comunidade se insere em um contexto histórico, socioeconômico e político mais amplo. Com isso, a pesquisa domiciliar permitiu a construção de significados voltados à SAN enquanto objeto de direito, e forneceu a base argumentativa para ações comunitárias voltadas ao setor público.

Em uma sociedade que ainda pouco reconhece alimentação enquanto direito humano, faz-se necessário enxergar a pesquisa em SAN para além da geração de conhecimento, como espaço de sua construção enquanto objeto de direito, e de constituição de sujeitos políticos que se reconhecem como portadores de direitos sociais.

É neste ponto que se insere a segunda implicação em se pensar a insegurança alimentar e nutricional dentro da abordagem da vulnerabilidade social, pois esta também diz respeito às possibilidades de enfrentamento. Em particular, o modelo de análise de vulnerabilidade social proposto por Kaztman ${ }^{46}$ incorpora o conjunto de recursos e estratégias que os indivíduos e famílias podem mobilizar para melhorar sua situação de bem-estar, evitar a deterioração de suas condições de vida ou diminuir sua vulnerabilidade. $\mathrm{O}$ enfrentamento da vulnerabilidade social vai além do quadro individual, como bem ressalta Porto, quando chama a atenção para "a importância de as populações ditas vulneráveis assumirem o seu papel de sujeitos coletivos ativos de transformação da sua própria condição de vulnerabilizados" ${ }^{\prime 7}$. Pensar a vulnerabilidade social implica em pensar a participação política e institucional dos indivíduos ${ }^{48}$, assim como exige situá-los como sujeitos políticos, pois segundo Porto et al..$^{45}$ é preciso:

[...] pensar a complexidade e conceitos como vulnerabilidade numa perspectiva crítica e emancipatória. Mais que atributos que definem pessoas e grupos mais ameaçados ou predispostos a problemas de saúde, em função de sua incapacidade de se defenderem, é importante analisar e intervir nos processos que vulnerabilizam os sujeitos. Isto significa superar a tendência a considerar tais populações como passivas e abstratas para situá-las como sujeitos políticos e históricos, culturalmente situados, detentores de direitos.

Neste sentido, qualquer pesquisa que toca a situação de vulnerabilidade torna-se instrumento em potencial da participação política dos sujeitos que se encontram na situação em questão.

$\mathrm{O}$ estudo mostrou que o acesso público a dados de interesse para a avaliação da situação de insegurança alimentar e nutricional é difícil. Difícil porque os dados estão desatualizados e incompletos, difícil pela complexidade dos indicadores e incoerência entre eles, e difícil porque os dados são agregados e não permitem captar a situação em áreas de tamanho menor. Os participantes do projeto, líderes comunitários, militam sem terem em mão dados tangíveis que possam apoiar os seus argumentos. A inserção destes líderes em conselhos, entre eles o distrital de saúde e o de moradores da subprefeitura, não facilitou, como poder-se-ia esperar, o acesso a tais dados.

Participar de pesquisa em qualidade outra do que a de informante não era prática dos membros da comunidade. A inserção nos processos de desenho da pesquisa, organização, análise e utilização dos resultados representou uma experiência totalmente nova para os participantes, conforme relataram. Apesar das dificuldades que podem representar a novidade desta experiência, os participantes tomaram plena parte do desenho da pesquisa e da sua organização, e utilizaram os seus resultados para a formulação de pautas de atuação, entre elas a formação de um conselho regional de segurança alimentar e nutricional, a reivindicação da implementação de um Restaurante Popular na região e um projeto de horta comunitária. Assim, de informantes se tornaram idealizadores e utilizadores da pesqui- 
sa, o que já representa um grande passo em direção ao controle de recursos que possam servir ao enfrentamento da vulnerabilidade social na qual se encontra a comunidade.

Antes de tudo, a experiência mostrou a relevância em se pensar a pesquisa em SAN como processo emancipatório, indissociável da ação e dos atores da transformação social. Essa perspectiva reconhece e dialoga com a necessidade de abordagens locais e qualitativas, que buscam compreender a insegurança alimentar e nutricional na sua dimensão cultural, como um processo inserido nas relações sociais e no cotidiano dos indivíduos, acarretando suas especificidades locais e a necessidade em se aproximar da subjetividade dos grupos sociais envolvidos. Porém, para além do local e do qualitativo, é a relevância de abordagens participativas o que mais se destaca nessa reflexão, pois o compreender e o agir são intrinsecamente ligados.

No mesmo movimento em que os resultados da pesquisa mostram o quanto a questão da SAN está inserida na da vulnerabilidade social, evidenciam o quão necessário se faz pensar a pesquisa em SAN como instrumento político e o conhecimento gerido como instrumento do poder. Na linha dos pensadores da pesquisa-ação, a avaliação da situação de SAN se constitui como processo emancipatório em si, pautado na necessidade do protagonismo dos membros da comunidade na construção do saber sobre a sua própria vulnerabilidade para poder enfrentá-la.

\section{Colaboradores}

MA Aliaga foi responsável pelo levantamento e análise dos dados, e pela escrita do artigo. MS Ribeiro contribuiu no levantamento e análise dos dados e escrita do artigo. SMC Santos contribuiu na coordenação dos processos de coleta e análise de dados e revisão do texto do artigo. LAB Trad foi responsável pela coordenação dos processos de coleta e análise dos dados, revisão e validação do artigo. 


\section{Referências}

1. Valente FLS. Do combate à fome à segurança alimentar e nutricional: o direito à alimentação adequada. In: Valente FLS. Direito humano à alimentação: desafios e conquistas. São Paulo: Cortez; 2002. p. 40-43.

2. Committee on World Food Security (CFS). Coming to terms with terminology: Food Security Nutrition Security Food Security and Nutrition Food and Nutrition Security. Rome: FAO; 2012.

3. Food and Agriculture Organization (FAO). Declaração de Roma Sobre a Segurança Alimentar Mundial e Plano de Ação da Cúpula Mundial da Alimentação. Rome: FAO; 1996.

4. Pérez-Escamilla R, Segall-Corrêa AM. Food insecurity measurement and indicators. Rev Nutr 2008; 21(Supl.):15-26.

5. Aliaga MA. Segurança alimentar e nutricional no contexto do desenvolvimento humano: análise comparativa de experiências internacionais de políticas públicas e avaliação [dissertação]. Salvador: Universidade Federal da Bahia; 2013.

6. Instituto Brasileiro de Geografia e Estatística (IBGE). Pesquisa Nacional por Amostra de Domicílios: Segurança Alimentar 2013. Rio de Janeiro: IBGE; 2014.

7. Instituto Brasileiro de Geografia e Estatística (IBGE). Pesquisa de Orçamentos Familiares 2008-2009: Antropometria e Estado Nutricional de Crianças, Adolescentes e Adultos no Brasil. Rio de Janeiro: IBGE; 2010.

8. Instituto Brasileiro de Geografia e Estatística (IBGE). Pesquisa de Orçamentos Familiares 2008-2009: Aquisição alimentar domiciliar per capita Brasil e Grandes Regiões. Rio de Janeiro: IBGE; 2010.

9. Instituto Brasileiro de Geografia e Estatística (IBGE). Pesquisa de Orçamentos Familiares 2008-2009: Avaliação nutricional da disponibilidade domiciliar de alimentos no Brasil. Rio de Janeiro: IBGE; 2010.

10. Instituto Brasileiro de Geografia e Estatística (IBGE). Pesquisa de Orçamentos Familiares 2008-2009: Análise do Consumo Alimentar Pessoal no Brasil. Rio de Janeiro: IBGE; 2011.

11. Godoy KC et al. Perfil e situação de insegurança alimentar dos usuários dos Restaurantes Populares no Brasil. Cad Saude Publica 2014; 30(6):1239-1249.

12. Lang RMF, Almeida CCB, Taddei JAAC. Segurança alimentar e nutricional de crianças menores de dois anos de famílias de trabalhadores rurais Sem Terra. Cien Saude Colet 2011; 16(7):3111-3118.

13. Oliveira JS, Israel P, Lira C, Augusta L, Sequeira DS, Filho MB. Anemia, hipovitaminose A e insegurança alimentar em crianças de municípios de Baixo Índice de Desenvolvimento Humano do Nordeste do Brasil. Rev Bras Epidemiol 2010; 13(4):651-664.

14. Pedraza DF, Gama JSFA. Segurança alimentar e nutricional de famílias com crianças menores de cinco anos do município de Campina Grande, Paraíba. Rev Bras Epidemiol 2015; 18(4):906-917.

15. Rocha EMB, Lima RT, Almeida PC. Insegurança alimentar relacionada à área de residência em município do Semiárido brasileiro. Cad Saúde Coletiva 2014; 22(2):205-211.

16. Santos LMP, Carneiro FF, Hoefel MGL, Santos W, Nogueira TQ. The precarious livelihood in waste dumps: A report on food insecurity and hunger among recyclable waste collectors. Rev Nutr 2013; 26(3):323-334.
17. Silva EKP, Medeiros DS, Martins PC, Sousa LA, Lima GP, Rêgo MAS, Silva TO, Freire AS, Silva FM. Insegurança alimentar em comunidades rurais no Nordeste brasileiro: faz diferença ser quilombola? Cad Saude Publica 2017; 33(4):1-14.

18. Souza MM, Pedraza DF, Menezes TN. Estado nutricional de crianças assistidas em creches e situação de (in) segurança alimentar de suas famílias. Cien Saude Colet 2012; 17(12):3425-3436.

19. Vianna RPT, Segall-Corrêa AM. Insegurança alimentar das famílias residentes em municípios do interior do estado da Paraíba, Brasil. Rev Nutr 2008; 21(Supl.):111-122.

20. Vieira VL, Souza MP, Cervato-Mancuso AM. Insegurança alimentar, vínculo mãe-filho e desnutrição infantil em área de alta vulnerabilidade social. Rev Bras Saúde Matern Infant 2010; 10(2):199-207.

21. Carvalho AS, Silva DO. Perspectivas de segurança alimentar e nutricional no Quilombo de Tijuaçu, Brasil: a produção da agricultura familiar para a alimentação escolar. Interface (Botucatu) 2014; 18(50):521-532.

22. Silva DO, Guerrero AFH, Guerrero CH, Toledo LM. A rede de causalidade da insegurança alimentar e nutricional de comunidades quilombolas com a construção da rodovia BR-163, Pará, Brasil. Rev Nutr 2008; 21(Supl.):83-97.

23. Panelli-Martins BE, Santos SMC, Assis AMO. Segurança alimentar e nutricional: desenvolvimento de indicadores e experimentação em um município da Bahia, Brasil. Rev Nutr 2008; 21(Supl.):65-81.

24. Brasil. Lei $\mathrm{n}^{\circ} 11.346$, de 15 de setembro de 2006. Cria o Sistema Nacional de Segurança Alimentar e Nutricional - SISAN com vistas em assegurar o direito humano à alimentação adequada e dá outras providências. Diário Oficial da União 2006; 15 set.

25. Assão TY, Cordeiro AA, Costa C, Cervato AM. Práticas e Percepções acerca da Segurança Alimentar e Nutricional entre os Representantes das Instituições Integrantes de um Centro de Referência localizado na Região do Butantã, Município de São Paulo. Saúde e Soc 2007; 16(1):102-116.

26. Burlandy L, Magalhães R, Lobato E, Florentino M. Potencialidades e limites para a implementação de sistemas locais de segurança alimentar e nutricional: estudo de caso em dois municípios do Estado do Rio de Janeiro. Brasília: MDS; 2014.

27. Costa CA, Bógus CM. Significados e Apropriações da Noção de Segurança Alimentar e Nutricional pelo Segmento da Sociedade Civil do Conselho Nacional de Segurança Alimentar e Nutricional. Saúde e Soc 2012; 21(1):12-22.

28. Gallina LS, Teo CRPA, Munaro PS, Oliveira VSH. Representações sobre Segurança Alimentar e Nutricional nos Discursos de um Conselho de Alimentação Escolar. Saúde e Soc 2012; 21(1):89-102.

29. Jacques ITO. A constitucionalização da alimentação: um direito a ser implementado adequadamente no Brasil [tese]. Porto Alegre: Pontifícia Universidade Católica do Rio Grande do Sul; 2014. 
30. Rocha NC, Doria NG, Boia JM, Bógus CM. Organização e dinâmica do Conselho Municipal de Segurança Alimentar e Nutricional de São Paulo: implicações para a sua atuação na construção da política municipal de Segurança Alimentar e Nutricional. Rev Nutr 2012; 25(1):133-146.

31. Cornwall A, Jewkes R. What is participatory research? Soc Sci Med 1995; 41(12):1667-1676.

32. Thiollent M. Metodologia da Pesquisa-ação. 14a ed. São Paulo: Cortez Editora; 2005.

33. Zuniga RB. La recherche-action et le contrôle du savoir. Int Rev Community Dev 1981; (5):35-44.

34. Salvador. Sistema de Informação Municipal (SIM) [Internet]. Indicadores. [2017 Ago 28]. Disponível em: http://www.sim.salvador.ba.gov.br/indicadores/

35. Pereira MHQ. Avaliação da segurança alimentar e nutricional: contribuições em âmbito municipal [dissertação]. Salvador: Universidade Federal da Bahia; 2014.

36. Salvador. Secretaria Municipal de Saúde (SMS) [Internet]. TABNET - Salvador. [acessado 2017 Ago 28]. Disponível em: http://www.tabnet.saude.salvador. ba.gov.br

37. Pereira DDA, Vieira VL, Fiore EG, Cervato-Mancuso AM. Insegurança Alimentar em Região de Alta Vulnerabilidade Social da Cidade de São Paulo. Segurança Aliment e Nutr 2006; 13(2):34-42.

38. Hoffmann R. Determinantes da Insegurança Alimentar no Brasil: Análise dos Dados da PNAD de 2004. Segurança Aliment e Nutr 2008; 15(1):49-61.

39. Pedraza DF. Grupos vulnerables y su caracterización como criterio de discriminación de la seguridad alimentaria y nutricional en Brasil. Rev Bras Saúde Matern Infant 2005; 5(3):367-375.

40. Kepple AW. O estado da segurança alimentar e nutricional no brasil: um retrato multidimensional. Brasília: FAO; 2014.

41. Brasil. Ministério da Saúde (MS). PNDS 2006: Pesquisa Nacional de Demografia e Saúde da Criança e da Mulher. Brasília: MS; 2008.
42. Vasconcelos EM. Educação popular e atenção à saúde da família. São Paulo: Hucitec; 1999.

43. Valente FLS. Fome, desnutrição e cidadania: inclusão social e direitos humanos. Saude e Soc 2003; 12(1):5160.

44. Freitas MCS, Pena PGL. Segurança alimentar e nutricional: A produção do conhecimento com ênfase nos aspectos da cultura. Rev Nutr 2007; 20(1):69-81.

45. Porto MFS, Rocha DF, Finamore R. Saúde coletiva, território e conflitos ambientais: bases para um enfoque socioambiental crítico. Cien Saude Colet 2014; 19(10):4071-4080.

46. Kaztman R. Notas sobre la medicion de la vulnerabilidad social. In: Kaztman R. Taller regional, la medicina de la pobreza, metodos y aplicaciones Borrador para discusión. México: BID, Birf, Cepal; 2000. p. 275-301.

47. Porto MFS. Complexidade, processos de vulnerabilização e justiça ambiental: um ensaio de epistemologia política. Rev Crit Cienc Sociais 2011; 93:31-58.

48. Muñoz Sánchez AI, Bertolozzi MR. Pode o conceito de vulnerabilidade apoiar a construção do conhecimento em Saúde Coletiva? Cien Saude Colet 2007; 12(2):319-324.

Artigo apresentado em 31/08/2018

Aprovado em 27/11/2018

Versão final apresentada em 29/11/2018 